\title{
Prevalencia de depresión en soldados regulares de un batallón de una ciudad colombiana, 2017
}

Sandra Milena Alvaran López a ; Sebastian Bedoya Mejía ${ }^{\text {b; }}$ Maria Ceneida Henao Valencia c; Juan Carlos Velasquez Correa ${ }^{c}$; Hugo Grisales Romero ${ }^{d}$

aTrabajadora social. Doctora en Cooperación para el Desarrollo. Universidad de Antioquia. sandra.alvaran@udea.edu.co

https://orcid.org/0000-0003-2036-6993

b Estudiante de Doctorado en Epidemiología y Bioestadística de la Universidad CES. 'Gerente en Sistemas de Información en Salud. Universidad de Antioquia 'Doctor en Epidemiología. Universidad de Antioquia

DOI 10.22517/25395203.21921

\section{Resumen}

Objetivo: Determinar la prevalencia de depresión y de sus dimensiones en soldados de un batallón colombiano.

Métodos: Estudio transversal en 410 soldados a quienes se les aplicó un cuestionario de depresión. Se calcularon las prevalencias para cada dominio y para la depresión en general y la asociación con factores de interés.

Resultados: La prevalencia de depresión fue del 8,8\%; las prevalencias de los dominios fueron: $11,7 \%$ para la autoestima negativa, $24,6 \%$ para la ideación suicida, $56,8 \%$ para pobre imagen social, $26,3 \%$ para afecto negativo, 23,4\% para desesperanza y 19,3\% para evitación.

Conclusión: Refinar los procedimientos de tamizaje en salud mental en el proceso de selección de los jóvenes soldados, para que sean desacuartelados aquellos aspirantes propensos a la depresión.

Palabras clave: Personal Militar, Síntomas Depresivos, Ideación Suicida, prevalencia, salud mental (Desc, 2019)
Depression prevalence in regular soldiers in a Colombian infantry battalion in 2017.

\begin{abstract}
Objective: To determine the prevalence of depression and its dimensions in soldiers in a Colombian battalion.

Method: This cross-sectional study was conducted to 410 soldiers; a questionnaire about depression issues was used to interview them.

The prevalence of depression was analyzed in general and specific terms. Addiotionally, some factors of interest that are associated with it were studied too.
\end{abstract}

Result: In general terms, the depression prevalence was $8,8 \%$. On the other hand, the prevalence of the depression dimensions was: $11,7 \%$ for negative self-esteem, $24,6 \%$ for suicidal thoughts, $56,8 \%$ for poor social image perception, $26,3 \%$ for negative affect, $23,4 \%$ for helplessness, and $19,3 \%$ for avoidance tendencies.

Conclusion: To conclude, it is necessary to improve the selection criteria in order to analyze the soldiers' mental health and, in this way, not to choose the candidates with depressive tendencies.

Key words: military personnel, depressive symptoms, suicidal ideation, prevalence, mental health (Desc, 2019)

\section{Introducción}

La Organización Mundial de la Salud ${ }_{(1)}$ considera que la depresión es un trastorno mental frecuente caracterizado por la presencia de tristeza, pérdida de interés o placer, sentimientos de culpa o falta de autoestima, trastornos del sueño o del apetito, sensación de cansancio y falta de concentración. Comprende un amplio espectro de trastornos que comparten ciertas características, varían en sus causas, intensidad, sintomatología, cronicidad curso e incluso en el comportamiento epidemiológico.

La depresión puede llegar a hacerse crónica o recurrente y dificultar sensiblemente el desempeño en el trabajo o la escuela y la capacidad para afrontar la vida diaria. Dependiendo del número y gravedad de los síntomas, un episodio depresivo se clasifica 
como leve, moderado o severo y distimia; esta última hace referencia a un tipo de trastorno de afecto o del estado de ánimo usualmente parecido a una forma menos severa de un trastorno depresivo, aunque en su forma más grave, puede conducir al suicidio. Si es leve, se puede tratar sin necesidad de medicamentos, pero cuando tiene carácter moderado o grave se pueden necesitar medicamentos y psicoterapia profesional (1) ${ }^{\circ}$

Además, la depresión también puede presentarse en personas con antecedentes de episodios maníacos, las cuales manifiestan un estado de ánimo exaltado y de mayor energía, lo que deriva en sobreactividad, habla atropellada o verborrea y menor necesidad de dormir; la depresión es un trastorno que se puede diagnosticar de forma fiable y que puede ser tratado por no especialistas en el ámbito de la atención primaria ${ }_{(2)}$.

La depresión puede afectar a cualquier persona sin importar sus condiciones sociales, económicas y puede coexistir con otras enfermedades severas; contribuyendo de manera significativa a la carga global de la enfermedad, especialmente por los altos niveles de discapacidad que ocasiona ${ }_{(3)}$.

La Encuesta Mundial de Salud Mental llevada a cabo en 17 países informó que en promedio 1 de cada 20 personas había tenido un episodio depresivo en el $2012_{(4)}$. Los trastornos depresivos comienzan a menudo a temprana edad, reducen el funcionamiento y frecuentemente son recurrentes. La necesidad de minimizar el flagelo de la depresión llevó a que la Asamblea de la Salud Mundial instara a la OMS y sus estados miembro a actuar en esta dirección ${ }_{(5)}$.

En cuanto a cifras estadísticas, en diferentes partes del mundo, se han reportado prevalencias entre el 8 y el $12 \%$ en la mayor parte de los países, por ejemplo, de un 3\% en Japón a un 16,9\% en los Estados Unidos ${ }_{(3)}$. En el 2015, los trastornos depresivos representaron un total de más de 50 millones de años vividos con discapacidad a escala mundial; más de $80 \%$ de esta carga de enfermedad no mortal ocurrió en países de ingresos bajos y medianos afectando a más de 300 millones de personas. Cada año se suicidan cerca de 800000 personas, siendo el suicidio la segunda causa de muerte en el grupo etario de 15 a 29 años; valga resaltar que el suicidio representa cerca de $1,5 \%$ de todas las defunciones en el mundo, por lo que se clasificó entre las 20 principales causas de muerte en el $2015_{(5)}$.

En Colombia, en el año 2003 se hizo la Encuesta Nacional de Salud Mental con 4544 adultos entre 18 y 65 años en 60 municipios del país; el 14,9\% refirió haber tenido síntomas de depresión a lo largo de la vida, con distribución irregular en el territorio nacional. En Bogotá se registró el 21,1\% de los casos, en la región central el 15,5\% y en la zona oriental el 8,1\% ${ }_{(6)}$. En un estudio realizado en el año 2015 por la misma entidad, el 5,7\% de los hombres encuestados presentaban ideación suicida. En la población universitaria de una institución castrense colombiana, la prevalencia del trastorno depresivo menor experimentado alguna vez en la vida fue del $10 \%$ y del $2 \%$ a un año, del $5 \%$ al $7 \%$ alguna vez en la vida para el trastorno depresivo mayor y del $2 \%$ al $7 \%$ en cuanto a prevalencia en curso para el año 2006 (7). En el contexto regional, a Colombia, según informe de la OMS, la prevalencia de depresión fue del $4,7 \%$ en contraste con Brasil con el 5,8\%, en Cuba con el 5,5\% y Paraguay con el 5,2\% ${ }_{(8)}{ }^{\circ}$

El Sistema Integral de Información de la Protección Social (SISPRO) reportó que las atenciones por depresión se han incrementado desde el año 2009, alcanzando para el año 2015 36584 atenciones ${ }_{(9)}$. En el estudio de estimación de la carga de enfermedad para Colombia realizado en 2010, se encontró que en las personas de 15 a 29 años la depresión mayor unipolar fue la primera causa de carga de la enfermedad con 168 años de vida saludable perdidos (Avisa) por 1.000 habitantes $_{(9)}$.

Cuando se particularizan los estudios de depresión en poblaciones específicas, como en el gremio castrense, Espitia Mancipe y Rincón Joya ${ }_{(10)}$, aplicaron la Escala de la Gravedad de Suicidio a 38507 reclutas que ingresaron al ejército en diferentes bases de entrenamiento entre abril de 2011 y noviembre de 2012 en Colombia, con el fin de examinar la asociación de factores sociodemográficos con la aparición de comportamientos suicidas. Los resultados de esta investigación sugieren que la mayoría de los soldados con planes e ideación suicida tuvieron los primeros inicios de estas conductas antes de su alistamiento en las Fuerzas Militares. En los últimos diecisiete años se han presentado 1155 casos de suicidio, un promedio de 67,9 casos de suicidios por año. Es de anotar que aun cuando se ha observado, en los últimos cuatro años una disminución del número promedio de suicidios, a 46,3 casos por año, las cifras siguen siendo alarmantes DGSM (2017). En el año 2016, en una población de 270.000 efectivos que correspondía al total de miembros de la Fuerza Pública se calculó una tasa de 14,74 suicidios por 100.000, tomando como referencia los 41 casos reportados ${ }_{(10)}$.

Desde la perspectiva anteriormente esbozada, el conocimiento de los indicadores de depresión en poblaciones específicas, como la que constituyen los soldados regulares, es una prioridad; por ello, este artículo refiere la prevalencia de la depresión en soldados regulares de un batallón colombiano en el año 2017, con el fin de que los entes encargados conozcan la magnitud de la problemática y establezcan estrategias que permitan una selección de personal con mejores condiciones de salud mental, a través de la utilización de instrumentos de tamizaje sensibles para la detección temprana de estas problemáticas.

\section{Materiales y métodos}

Se trata de un estudio de corte transversal donde se estimó la prevalencia de depresión en 410 soldados regulares de un batallón de una ciudad colombiana. Se aplicó un cuestionario de depresión para hombres $(\mathrm{CDH})$ elaborado por Álvarez y Londoño, el cual fue validado por Londoño y Wenceslao en España ${ }_{(11)}$. Este instrumento está compuesto por 40 ítems que evalúan síntomas considerados propios de la depresión en hombres, con opciones de respuesta en escala likert de 
1 a 4 , donde 1 representa «nunca», 2 «casi nunca», 3 «casi siempre» y 4 «siempre». La escala evalúa 6 dimensiones: autoimagen negativa, ideación suicida, pobre imagen social, afecto negativo, desesperanza y evitación. Para obtener el puntaje de depresión general se suman los puntajes obtenidos en todos los ítems, obteniéndose de 1 a 85, que indica ausencia de depresión, de 86 a 105 síntomas leves/estado subclínico, de 106 a 125 depresión moderada y de 126 a 140 depresión severa.

En el análisis de la información se realizó, primero, una descripción de las variables constitutivas de cada dimensión; luego se estimó la prevalencia de la depresión la cual se acompañó del intervalo de confianza del 95\%. Se conformaron dos grupos, uno constituido por aquellos soldados que el instrumento detectó con depresión y el otro por aquellos que se identificaron sin depresión, tendiente a explorar la asociación con variables de interés utilizando como medida de la asociación la razón de prevalencias, RP, cuando las variables independientes fueran cualitativas. Se acogieron los principios éticos recogidos en la declaración de Helsinki y se obtuvo el informe favorable del comité de ética y del área de psicología militar del batallón. Todos los soldados firmaron el documento de consentimiento informado específico, siguiendo los cánones formalizados en la investigación con humanos. Para la obtención de los resultados, se acudió a los programas estadísticos SPSS, v.23 y Stata, v.15; el office fue la herramienta para la elaboración del informe final.

\section{Resultados}

Se aplicó el instrumento a 410 soldados regulares adscritos a un batallón de una ciudad colombiana en el año 2017, de los cuales el 52,6\% provenían del departamento de Antioquia; el $65,5 \%$ de ellos tenían más de 20 años; el 42,4\% respondió que tenía estudios secundarios, aunque es importante advertir que en el 38,5\% de los casos se desconoció el nivel de estudios de los soldados.

En general, las preguntas que constituyen cada uno de los dominios del instrumento que miden la depresión, están formuladas en sentido negativo con respuestas en escala tipo Likert (Nunca, Casi nunca, Casi siempre y Siempre). Se observó que en las respuestas a todos los interrogantes las frecuencias predominantes fueron entre nunca y casi nunca, aunque, en particular, se observaron frecuencias de respuestas que llamaron la atención: cuando se indagó acerca de la autoimagen negativa que tenían los soldados con base en las preguntas para tal fin del instrumento, se destacó que el $12,4 \%$ pensaban que los demás estaban mejor sin ellos; en la valoración de la ideación suicida el $9 \%$ respondió que entre casi siempre o siempre fantasea con dormir y no despertar; en lo que se relaciona con la valoración que hicieron los soldados de la imagen social, llamó la atención que casi siempre o siempre el $21,5 \%$ y $16,3 \%$ han sentido que otros los ven como apagados y que se sienten insatisfechos con lo que hacían, respectivamente; en la dimensión afecto negativo, se resaltó que la ansiedad, el agotamiento y la intranquilidad tuvieron frecuencias de presentación importantes (con respuestas de casi siempre y siempre en el $28,3 \%, 26,8 \%$ y $25,2 \%$, en ese orden). En general, con respecto a la dimensión de desesperanza, el 42,2\% no imagina cómo será su vida dentro de 10 años; en las respuestas de los soldados en el tópico relacionado con la evitación, el 56,4\% entre casi siempre y siempre refirió que buscaba estar ocupado para no pensar. De igual manera, el 47,8\% respondió que es mejor evadir dificultades haciendo otras actividades con sus amigos: 
Tabla 1. Frecuencias de las respuestas a los interrogantes que constituyen cada una de las dimensiones para la medición de la depresión ( $\mathrm{n}=\mathbf{4 1 0})$ de los soldados de una ciudad de Colombia, 2017.

\begin{tabular}{|c|c|c|c|c|}
\hline \multirow[t]{2}{*}{ Dimensiones / preguntas } & Nunca & Casi nunca & $\begin{array}{c}\text { Casi } \\
\text { siempre }\end{array}$ & $\begin{array}{c}\text { Siemp } \\
\text { re }\end{array}$ \\
\hline & n (\%) & n(\%) & $\mathbf{n}(\%)$ & $\mathrm{n}(\%)$ \\
\hline \multicolumn{5}{|l|}{ Autoestima negativa } \\
\hline ¿Suele desmeritar lo que hace? & $\begin{array}{c}328 \\
(80,0)\end{array}$ & $43(10,5)$ & $29(7,1)$ & $\begin{array}{c}10 \\
(2,4)\end{array}$ \\
\hline $\begin{array}{l}\text { ¿Ha pensado que los demás estarian } \\
\text { mejor sin usted? }\end{array}$ & $\begin{array}{c}309 \\
(75,4)\end{array}$ & $50(12,2)$ & $34(8,3)$ & $\begin{array}{c}17 \\
(4,1)\end{array}$ \\
\hline ¿Ha sentido pesar de si mismo? & $\begin{array}{l}312 \\
(76,1)\end{array}$ & $63(15,4)$ & $22(5,4)$ & $\begin{array}{c}13 \\
(3,2)\end{array}$ \\
\hline $\begin{array}{l}\text { ¿Usa palabras cómo incapaz e inútil } \\
\text { para definirse? }\end{array}$ & $\begin{array}{c}358 \\
(87,3)\end{array}$ & $30(7,3)$ & $17(4,1)$ & $5(1,2)$ \\
\hline \multicolumn{5}{|l|}{ Ideación suicida } \\
\hline $\begin{array}{l}\text { ¿Ha deseado terminar con su vida } \\
\text { para no sufrir más? }\end{array}$ & $\begin{array}{c}372 \\
(90,7)\end{array}$ & $23(5,6)$ & $11(2,7)$ & $4(1,0)$ \\
\hline ¿Fantasea con dormir y no despertar? & $\begin{array}{c}336 \\
(82,0)\end{array}$ & $37(9,0)$ & $28(6,8)$ & $9(2,2)$ \\
\hline $\begin{array}{l}\text { ¿Ha pensado en formas para terminar } \\
\text { con su vida? }\end{array}$ & $\begin{array}{c}369 \\
(90,0)\end{array}$ & $27(6,6)$ & $10(2,4)$ & $4(1,0)$ \\
\hline $\begin{array}{l}\text { ¿Ha deseado morir para solucionar } \\
\text { sus problemas? }\end{array}$ & $\begin{array}{c}369 \\
(90,0)\end{array}$ & $23(5,6)$ & $15(3,7)$ & $3(, 7)$ \\
\hline \multicolumn{5}{|l|}{ Pobre imagen social } \\
\hline $\begin{array}{l}\text { ¿Ha sentido que otros lo ven como } \\
\text { pesimista? }\end{array}$ & $\begin{array}{c}321 \\
(78,3)\end{array}$ & $59(14,4)$ & $25(6,1)$ & $5(1,2)$ \\
\hline ¿Se siente frustrado? & $\begin{array}{c}300 \\
(73,2)\end{array}$ & $52(12,7)$ & $38(9,3)$ & $\begin{array}{c}20 \\
(4,9)\end{array}$ \\
\hline $\begin{array}{l}\text { ¿Siente insatisfacción con lo que } \\
\text { hace? }\end{array}$ & $\begin{array}{c}300 \\
(73,2)\end{array}$ & $43(10,5)$ & $46(11,2)$ & $\begin{array}{c}21 \\
(5,1)\end{array}$ \\
\hline $\begin{array}{l}\text { ¿Se comporta de tal manera que los } \\
\text { demás piensan que parece otra } \\
\text { persona? }\end{array}$ & $\begin{array}{c}295 \\
(72,0)\end{array}$ & $52(12,7)$ & $38(9,3)$ & $\begin{array}{c}25 \\
(6,1)\end{array}$ \\
\hline $\begin{array}{l}\text { Le han dicho que es una persona } \\
\text { dificil de tratar? }\end{array}$ & $\begin{array}{c}283 \\
(69,0)\end{array}$ & $74(18,0)$ & $40(9,8)$ & $\begin{array}{c}13 \\
(3,2)\end{array}$ \\
\hline $\begin{array}{l}\text { ¿Ha sentido que otros lo ven como } \\
\text { apagado? }\end{array}$ & $\begin{array}{c}227 \\
(55,4)\end{array}$ & $95(23,2)$ & $65(15,9)$ & $\begin{array}{c}23 \\
(5,6)\end{array}$ \\
\hline
\end{tabular}

\begin{tabular}{|c|c|c|c|c|}
\hline \multirow[t]{2}{*}{ Dimensiones / preguntas } & Nunca & Casi nunca & $\begin{array}{c}\text { Casi } \\
\text { siempre }\end{array}$ & $\begin{array}{c}\text { Siemp } \\
\text { re }\end{array}$ \\
\hline & n (\%) & $\mathrm{n}(\%)$ & $\mathbf{n}(\%)$ & $\mathbf{n}(\%)$ \\
\hline \multicolumn{5}{|l|}{ Afecto negativo } \\
\hline ¿Se siente agotado? & $\begin{array}{c}142 \\
(34,6)\end{array}$ & $158(38,5)$ & $85(20,7)$ & $\begin{array}{l}25 \\
(6,1)\end{array}$ \\
\hline ¿Se siente vacío? & $\begin{array}{c}272 \\
(66,3)\end{array}$ & $72(17,6)$ & $45(11,0)$ & $\begin{array}{c}21 \\
(5,1)\end{array}$ \\
\hline $\begin{array}{l}\text { ¿Tiene dificultad para controlar sus } \\
\text { emociones? }\end{array}$ & $\begin{array}{c}235 \\
(57,3)\end{array}$ & $80(19,5)$ & $61(14,9)$ & $\begin{array}{c}34 \\
(8,3)\end{array}$ \\
\hline${ }_{i}$ Se siente inquieto & $\begin{array}{c}240 \\
(58,5)\end{array}$ & $80(19,5)$ & $64(15,6)$ & $\begin{array}{l}26 \\
(6,3)\end{array}$ \\
\hline ¿Se siente irritable? & $\begin{array}{c}321 \\
(78,3)\end{array}$ & $57(13,9)$ & $28(6,8)$ & $4(1,0)$ \\
\hline $\begin{array}{l}\text { ¿Se ha sentido intranquilo } \\
\text { especialmente en la mañana? }\end{array}$ & $\begin{array}{l}224 \\
(54,6)\end{array}$ & $82(20,0)$ & $68(16,6)$ & $\begin{array}{c}36 \\
(8,8)\end{array}$ \\
\hline ¿Ha deseado abandonar todo? & $\begin{array}{c}322 \\
(78,5)\end{array}$ & $43(10,5)$ & $33(8,0)$ & $\begin{array}{c}12 \\
(2,9)\end{array}$ \\
\hline $\begin{array}{l}\text { ¿Se siente insatisfecho con lo que ha } \\
\text { hecho de su vida? }\end{array}$ & $\begin{array}{c}299 \\
(72,9)\end{array}$ & $53(12,9)$ & $27(6,6)$ & $\begin{array}{c}31 \\
(7,6)\end{array}$ \\
\hline $\begin{array}{l}\text { ¿Reacciona agresivo ante situaciones } \\
\text { normales? }\end{array}$ & $\begin{array}{c}318 \\
(77,6)\end{array}$ & $64(15,6)$ & $20(4,9)$ & $8(2,0)$ \\
\hline ¿Se siente estresado? & $\begin{array}{c}208 \\
(50,7)\end{array}$ & $119(29,0)$ & $60(14,6)$ & $\begin{array}{c}23 \\
(5,6)\end{array}$ \\
\hline ¿Se siente cansado sin razón & $\begin{array}{c}292 \\
(71,2)\end{array}$ & $49(12,0)$ & $52(12,7)$ & $\begin{array}{c}17 \\
(4,1)\end{array}$ \\
\hline ¿Se siente impaciente? & $\begin{array}{c}272 \\
(66,3)\end{array}$ & $79(19,3)$ & $45(11,0)$ & $\begin{array}{c}14 \\
(3,4)\end{array}$ \\
\hline $\begin{array}{l}\text { ¿Se ha sentido ansioso especialmente } \\
\text { en la mañana? }\end{array}$ & $\begin{array}{c}229 \\
(55,9)\end{array}$ & $65(15,9)$ & $66(16,1)$ & $\begin{array}{l}50(12, \\
2)\end{array}$ \\
\hline ¿Se siente que no sirve para nada? & $\begin{array}{c}370 \\
(90,2)\end{array}$ & $22(5,4)$ & $14(3,4)$ & $4(1,0)$ \\
\hline $\begin{array}{l}\text { ¿Ha tenido cambio brusco en su } \\
\text { estado de ánimo? }\end{array}$ & $\begin{array}{c}244 \\
(59,5)\end{array}$ & $87(21,2)$ & $63(15,4)$ & $\begin{array}{c}16 \\
(3,9)\end{array}$ \\
\hline \multicolumn{5}{|l|}{ Desesperanza } \\
\hline $\begin{array}{l}\text { ¿Ha sentido que tiene pocas opciones } \\
\text { de realizarse como persona en la } \\
\text { vida? }\end{array}$ & $\begin{array}{c}270 \\
(65,9)\end{array}$ & $73(17,8)$ & $42(10,2)$ & $\begin{array}{c}25 \\
(6,1)\end{array}$ \\
\hline $\begin{array}{l}\text { ¿No imagina cómo será su vida } \\
\text { dentro de } 10 \text { años? }\end{array}$ & $\begin{array}{l}155(37, \\
8)\end{array}$ & - & $111(27,1)$ & $\begin{array}{l}144(3 \\
5,1)\end{array}$ \\
\hline $\begin{array}{l}\text { ¿Presiente que su futuro va a ser más } \\
\text { malo que bueno? }\end{array}$ & $\begin{array}{c}350 \\
(85,4)\end{array}$ & $39(9,5)$ & $14(3,4)$ & $7(1,7)$ \\
\hline \multicolumn{5}{|l|}{ Evitación } \\
\hline ¿Tiene problemas para dormir bien? & $\begin{array}{c}314 \\
(76,6)\end{array}$ & $49(12,0)$ & $27(6,6)$ & $\begin{array}{c}20 \\
(4,9)\end{array}$ \\
\hline $\begin{array}{l}\text { ¿Ha tenido relaciones de pareja } \\
\text { paralelas? }\end{array}$ & $\begin{array}{c}268 \\
(65,4)\end{array}$ & $41(10,0)$ & $77(18,8)$ & $\begin{array}{c}24 \\
(5,9)\end{array}$ \\
\hline \multirow[t]{2}{*}{ Dimensiones / preguntas } & Nunca & Casi nunca & $\begin{array}{c}\text { Casi } \\
\text { siempre }\end{array}$ & $\begin{array}{l}\text { Siemp } \\
\text { re }\end{array}$ \\
\hline & n (\%) & $\mathrm{n}(\%)$ & $\mathbf{n}(\%)$ & $\mathrm{n}(\%)$ \\
\hline $\begin{array}{l}\text { ¿Ha buscado experimentar } \\
\text { sensaciones extremas aun cuando } \\
\text { ponga en riesgo su vida? }\end{array}$ & $\begin{array}{c}249 \\
(60,7)\end{array}$ & $68(16,6)$ & $55(13,4)$ & $\begin{array}{c}38 \\
(9,3)\end{array}$ \\
\hline $\begin{array}{l}\text { ¿Se le dificulta tomar decisiones } \\
\text { cotidianas? }\end{array}$ & $\begin{array}{c}277 \\
(67,6)\end{array}$ & $77(18,8)$ & $42(10,2)$ & $\begin{array}{c}14 \\
(3,4)\end{array}$ \\
\hline $\begin{array}{l}\text { ¿Busca estar ocupado para no } \\
\text { pensar? }\end{array}$ & $\begin{array}{c}114 \\
(27,8)\end{array}$ & $65(15,9)$ & $129(31,5)$ & $\begin{array}{c}102 \\
(24,9)\end{array}$ \\
\hline $\begin{array}{l}\text { ¿Siente que es mejor evadir } \\
\text { dificultades haciendo otras } \\
\text { actividades con sus amigos? }\end{array}$ & $\begin{array}{c}160 \\
(39,0)\end{array}$ & $54(13,2)$ & $91(22,2)$ & $\begin{array}{c}105 \\
(25,6)\end{array}$ \\
\hline $\begin{array}{l}\text { ¿Bebe sin poder parar, hasta } \\
\text { embriagarse? }\end{array}$ & $\begin{array}{l}276 \\
(67,3)\end{array}$ & $84(20,5)$ & $36(8,8)$ & $\begin{array}{c}14 \\
(3,4)\end{array}$ \\
\hline $\begin{array}{l}\text { ¿Se queja para conmover a otros y } \\
\text { obtener apoyo? }\end{array}$ & $\begin{array}{c}336 \\
(82,0)\end{array}$ & $49(12,0)$ & $18(4,4)$ & $7(1,7)$ \\
\hline
\end{tabular}


Cuando se consideraron los puntajes promedio obtenidos en cada una de las dimensiones, el correspondiente al afecto negativo fue el que sobresalió (promedio=23,6 puntos $\mathrm{DE}=8,0$ puntos) seguido por los puntajes promedio de evitación (Promedio $=13,9$ puntos $\mathrm{DE}=3,8$ puntos) y pobre imagen social (Promedio=8,9 puntos $\mathrm{DE}=3,3$ puntos). Las menores puntuaciones promedio se obtuvieron en las dimensiones de autoimagen negativa (Promedio $=5,3$ puntos $\mathrm{DE}=2,1$ puntos) e ideación suicida (Promedio=4,7 puntos $\mathrm{DE}=1,8$ puntos) respectivamente. Figura 1:

Figura 1. Promedio del puntaje en cada dimensión de la depresión de cada uno de los soldados pertenecientes a un batallón de una ciudad colombiana, 2017.

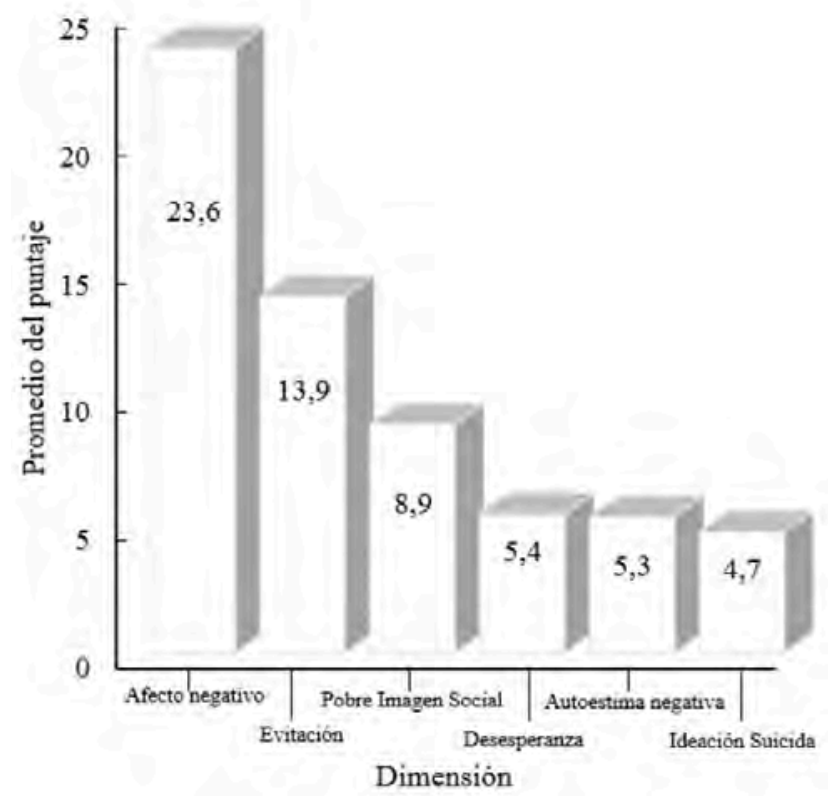

Según las condiciones de interpretación de las seis dimensiones que componen el $\mathrm{CDH}$, la autoestima positiva tuvo la mayor valoración, en el 88,3\% de los casos. Es llamativo el hecho de que del $23,9 \%$ de los soldados que tuvieron algún pensamiento relacionado con el suicidio, el 3,2\% de esos soldados lo tuvieron en mayor proporción. Aproximadamente, por cada soldado con alto nivel en la valoración de la pobre imagen social, se encontraron tres con valoración baja. Relación que fue de 7 a 1, 45 a 1 y 67 a 1 , con respecto a las dimensiones de afecto negativo, desesperanza y evitación, respectivamente. Se concluye que las prevalencias de cada uno de los dominios constitutivos del cuestionario para depresión en hombres fueron del 11,7\% para la autoestima negativa (IC 95\%: 8,5\% 14,9\%), del 23,9\% para la ideación suicida (IC 95\%: 19,7\% 28,2\%), del 56,8\% para pobre imagen social (IC 95\%: $51,9 \%$ $61,7 \%$ ), del $26,3 \%$ para afecto negativo (IC 95\%: $22,0 \%$ $30,7 \%$ ), del $23,4 \%$ para desesperanza (IC 95\%: $19,2 \%$ 27,6\%) y del 19,3\% para evitación (IC 95\%: 15,3\% 23,2\%):
Tabla 2. Frecuencias de las categorías de interpretación de cada dimensión de los soldados pertenecientes a un batallón a una ciudad de Colombia, 2017.

\begin{tabular}{|c|c|c|c|c|}
\hline \multirow{2}{*}{ Dimensión } & \multirow{2}{*}{$\mathbf{n}$} & \multirow{2}{*}{$\%$} & \multicolumn{2}{|c|}{ IC $(95 \% \text { para p* })^{* *}$} \\
\hline & & & Inferior & Superior \\
\hline \multicolumn{5}{|c|}{ Autoestima negativa } \\
\hline Ausencia & 362 & 88,3 & 85,1 & 91,5 \\
\hline Moderada & 48 & 11,7 & 8,5 & 14,9 \\
\hline \multicolumn{5}{|l|}{ Ideación Suicida } \\
\hline Ausencia & 312 & 76,1 & 71,8 & 80,3 \\
\hline $\begin{array}{l}\text { Pensamientos de } \\
\text { muerte }\end{array}$ & 71 & 17,3 & 13,5 & 21,1 \\
\hline Ideación suicida & 14 & 3,4 & 1,5 & 5,3 \\
\hline Alta ideación & 13 & 3,2 & 1,4 & 5 \\
\hline \multicolumn{5}{|c|}{ Pobre Imagen Social } \\
\hline Bajo nivel & 177 & 43,2 & 38,3 & 48,1 \\
\hline Nivel medio & 179 & 43,7 & 38,7 & 48,6 \\
\hline Alto nivel & 54 & 13,2 & 9,8 & 16,6 \\
\hline \multicolumn{5}{|l|}{ Afecto negativo } \\
\hline Bajo nivel & 302 & 73,7 & 69,3 & 78 \\
\hline Nivel medio & 65 & 15,9 & 12,2 & 19,5 \\
\hline Alto nivel & 43 & 10,5 & 7,4 & 13,6 \\
\hline \multicolumn{5}{|l|}{ Desesperanza } \\
\hline Bajo nivel & 314 & 76,6 & 72,4 & 80,8 \\
\hline Nivel medio & 89 & 21,7 & 17,6 & 25,8 \\
\hline Alto nivel & 7 & 1,7 & 0,3 & 3,1 \\
\hline \multicolumn{5}{|l|}{ Evitación } \\
\hline Bajo nivel & 331 & 80,7 & 76,8 & 84,7 \\
\hline Nivel medio & 74 & 18 & 14,2 & 21,9 \\
\hline Alto nivel & 5 & 1,2 & 0,4 & 2,8 \\
\hline
\end{tabular}

*p es la proporción poblacional de la categoría de interés de cada dimensión; **Intervalo de confianza binomial exacto

Según las condiciones de interpretación del $\mathrm{CDH}$, la prevalencia de depresión en los soldados regulares fue del 8,8\% (IC 95: 5,9\% 11,6\%). De todos los niveles de depresión, fue más prevalente el de síntomas leves/estado subclínico con el 5,9\% (IC 95\%: $3,5 \%$ 6,2\%). En general, por cada soldado con depresión severa, se presentaron aproximadamente 125 sin depresión: 
Figura 2. Niveles de depresión de los militares de un batallón de un departamento de Colombia, 2017

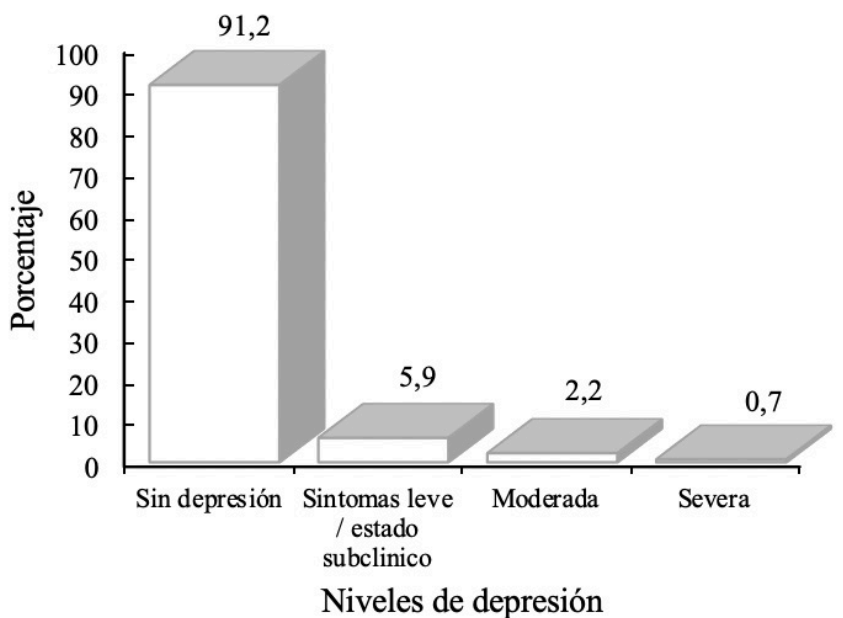

Para cada una de las dimensiones, se consideraron las categorías que expresaban una valoración negativa. Especificamente, para la autoestima negativa se seleccionó la categoría moderada. Para la ideación suicida, las categorías pensamientos de muerte, ideación suicida y alta ideación, las cuales se agruparon en una que refiriera algún asomo de ideación suicida y así sucesivamente. Se hizo con el fin de mostrar la distribución del número de dimensiones en las cuales los soldados reflejaron problemáticas. Se encontró que el $33,2 \%$ de los soldados (136) tuvieron puntuaciones que indicaban situaciones anómalas en una de las dimensiones seguida del 25,9\% (106 soldados) cuyas puntuaciones reflejaron situaciones problemáticas en dos dimensiones; en el $86,1 \%$ tuvieron puntuaciones negativas en máximo cuatro dimensiones y el 5,4\% en todas:

Figura 3. Frecuencia del número de dimensiones en las cuales los soldados reflejaron problemáticas de acuerdo con sus respuestas.

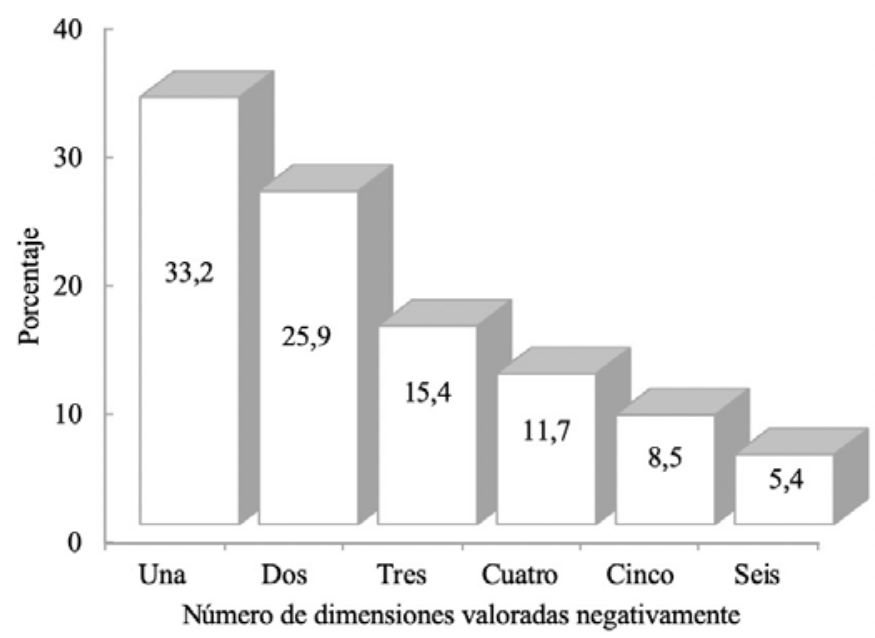

Cuando se consideró la distribución conjunta de cada una las características de interés de los soldados regulares, descritas anteriormente, con respecto a la depresión, se encontró que esta fue más prevalente en los soldados regulares que tenían entre 18 y 19 años, con una oportunidad estimada de la presentación de la misma de 1,5 veces con respecto a los soldados que tenían entre 20 y 26 años, aunque las diferencias no fueron significativas. De igual manera, la prevalencia de depresión fue mayor en los soldados que provenían del departamento de Antioquia, en relación con los que tenían su asiento en el departamento de Córdoba o en otros departamentos, aunque la diferencia no tuvo significación estadística. Con respecto al nivel educativo, la prevalencia mayor de depresión se conoció en los soldados regulares que tenían como máximo nivel educativo la básica primaria, aunque las diferencias no fueron significativas con respeto los soldados que tenían estudios secundarios:

Tabla 3. Distribución de frecuencias de algunos factores demográficos según el diagnóstico de depresión y valoración de la relación en soldados regulares de un batallón colombiano, 2017

\begin{tabular}{|c|c|c|c|c|c|c|c|c|}
\hline \multirow{3}{*}{ Características } & \multicolumn{4}{|c|}{ Depresión } & \multirow{3}{*}{$\mathbf{p}^{*}$} & \multirow{3}{*}{$\mathbf{R P}$} & \multirow{2}{*}{\multicolumn{2}{|c|}{ IC (95\% RP) }} \\
\hline & \multicolumn{2}{|c|}{$\mathrm{Si}$} & \multicolumn{2}{|c|}{ No } & & & & \\
\hline & $\mathbf{n}$ & $\%$ & $\mathbf{n}$ & $\%$ & & & Inferior & Superior \\
\hline \multicolumn{9}{|l|}{ Edad } \\
\hline 18-19 años & 16 & 11,3 & 126 & 88,7 & \multirow{2}{*}{0,195} & \multirow{2}{*}{1,5} & \multirow{2}{*}{0,8} & \multirow{2}{*}{2,8} \\
\hline $20-26$ años* & 20 & 7,5 & 248 & 92,5 & & & & \\
\hline \multicolumn{9}{|c|}{ Departamento de procedencia } \\
\hline Antioquia & 22 & 10,8 & 181 & 89,2 & 0,230 & 1,6 & 0,7 & 3,3 \\
\hline Otros & 5 & 6,5 & 72 & 93,5 & 0,905 & 0,9 & 0,3 & 2,7 \\
\hline Córdoba* & 9 & 6,9 & 121 & 93,1 & & & & \\
\hline \multicolumn{9}{|l|}{ Nivel educativo } \\
\hline Primaria & 6 & 18,8 & 26 & 81,3 & 0,085 & 2,1 & 0,9 & 4,9 \\
\hline Otros & 11 & 6,7 & 153 & 93,3 & 0,439 & 0,8 & 0,4 & 1,5 \\
\hline Secundaria* & 19 & 8,9 & 195 & 91,1 & & & & \\
\hline
\end{tabular}

\section{Discusión}

La depresión es uno de los problemas que en mayor medida atenta contra la salud mental de la población. Dependiendo de las actividades que cada grupo poblacional realiza, su frecuencia puede variar. De acuerdo con datos de la OMS, se ha estimado que 450 millones de personas a nivel mundial padecen alguna forma de trastorno mental y de ellas, entre el 27 y $33 \%$ padecen depresión. Se estima que la depresión puede llegar a ser la segunda causa de discapacidad a nivel mundial en el 2020 y se convertirá en un problema de salud pública ${ }_{(12)}$.

En particular, el personal adscrito a las fuerzas militares, especialmente los de menor rango, los soldados rasos, han sido considerados como una población altamente expuesta a problemas de salud mental, especialmente por la exigencia del cumplimiento de los postulados en que se basa su filosofía, en términos de disciplina, alcance, logros y discreción.

La presencia de problemas de salud mental en el personal militar trae consigo consecuencias sobre el rendimiento y mantenimiento en servicio activo. Al respecto, Miralles 
identificó que los militares presentaban una amplia gama polisintomática que comprendía dificultades para relacionarse, delirios de persecución, hipocondriasis, problemas de atención y síntomas graves de ansiedad y depresión. En un estudio realizado a los excombatientes de la guerra del Golfo, se registró, en la mitad de ellos, la presencia de depresión, caracterizado por pensamientos intrusivos y estados de hipervigilancia, entre otros $(13)$.

En Colombia, son pocas las investigaciones sobre salud mental que se conocen en relación con los soldados activos y ex militares. En cambio, en otros países donde se ha hecho mayor investigación al respecto, los resultados indican que uno de los trastornos mentales comúnmente asociados a los combatientes y excombatientes es el estrés postraumático, comórbido con el trastorno depresivo mayor y con la ansiedad generalizada $_{(14)}$.

$\mathrm{Al}$ respecto, Adler et al. ${ }_{(15)}$ analizaron los síntomas de estrés postraumático y depresión en 2114 soldados y encontraron que la participación en operaciones de despliegue más largas y las primeras operaciones en su historia de vida se relacionan con la depresión ${ }_{(15)}$. Al indagar acerca de los problemas de salud mental asociados a la exposición en las operaciones de despliegue, se encontró que los reservistas del Reino Unido que participaron en la guerra de Irak del 2003 reportaron experiencias traumáticas y dificultades en la adaptación al regresar al hogar ${ }_{(16)}$

La depresión es un problema grave que afecta la salud mental del personal militar tanto cuando están activos como cuando terminan su periplo en la milicia; así se evidencia en la problemática indicada por militares después de la primera y segunda guerra mundial, en un estudio en 1871 veteranos de la guerra australiana -que habían participado de la guerra del golfo pérsico -donde se encontró que el 9\% presentó depresión (17) ${ }^{\circ}$

La investigación que realizamos presenta la experiencia de la depresión en un contingente de 410 soldados regulares adscritos a un batallón colombiano. A continuación, se comparan los datos obtenidos en la presente investigación con datos de otras investigaciones, en relación con las siguientes variables: a) procedencia; b) nivel de escolaridad; c) la prevalencia de depresión; d) proporción de desesperanza e ideación suicida:

a. Procedencia: en el presente estudio el $52,6 \%$ de los soldados rasos provenían del departamento de Antioquia y el 34,6\% tenían entre 18 y 19 años. En un estudio realizado en militares regulares de primer año que representan la totalidad de un contingente particular, el $41,0 \%$ provenían de la región Caribe (18) (19), lo que sugiere que el personal que vive en la región se enlista en el batallón más cercano.

b. Nivel de escolaridad: El máximo nivel de escolaridad de los soldados fue estudios secundarios, con el 42,4\%. Nivel educativo que en Ecuador, en el año 2007, en la Escuela de Inteligencia Militar de las Fuerzas Armadas, alcanzó el 72,7\% (20).

c. Prevalencia de depresión: En este estudio fue del $8,8 \%$ donde el $5,9 \%$ fue leve y el $2,2 \%$ moderada. Es importante indicar que si la depresión es leve, se puede tratar sin necesidad de medicamentos, pero cuando tiene carácter moderado o grave se precisan medicamentos y psicoterapia profesional (1). Los indicadores hallados en este estudio están muy por debajo de una prevalencia del $51,8 \%$, leve en el $95,4 \%$ y moderada en el 4,6\%, de acuerdo con Moreno ${ }_{(20)}$, en milicianos ecuatorianos que aún no estaban en servicio activo. Por otro lado, en un estudio en tropas de combate que regresaron de Afganistán, la depresión varió entre el 7,1\% a 7,9\%. Grieger et al. (21) demostraron en un estudio realizado a soldados que presentaban heridas físicas, que el $4,4 \%$ tenía depresión al primer mes, el 8,9\% a los cuatro meses y $9,3 \%$ a los siete meses ${ }_{(21)}$, lo que reitera que la depresión puede aumentar en relación con las secuelas físicas y las variables de exposición al combate.

\section{d. Proporción de desesperanza e ideación suicida:}

En este estudio, la proporción de desesperanza fue de 23,4\% y del 24,6\% para la ideación suicida . Al respecto, Abello et al. ${ }_{(22)}$, en un estudio en un grupo de jóvenes de una escuela militar colombiana, de la región Caribe (41\%) y el centro del país (12\%), encontraron correlación significativa entre la desesperanza- depresión y depresión - riesgo suicida (22) ; la ideación suicida estimada fue el 4,7\% (22), hallazgo que contrasta con los resultados de una autopsia psicológica basada en consultas sobre la muerte de todos los soldados varones de 18 a 21 años que prestaron servicio en el ejército israelí y murieron por suicidio entre 2009 y 2013 , del $51,6 \%$, donde los soldados con depresión habían mostrado más signos de planificación del acto ${ }_{(23)}$ reportaron que la ideación suicida está relacionada con la depresión, en un estudio en 414 soldados por primera vez en Corea entre los años 2014 y 2015 (24).

En conclusión, tanto los resultados obtenidos como las observaciones de trabajos internacionales enfatizan la importancia de realizar evaluaciones rutinarias y periódicas de la salud mental del personal militar, en general, y de los soldados rasos en particular, especialmente de la sintomatología depresiva, antes y después de la inserción en la vida militar dado que su carácter constitucional determina una misión 
específica que requiere especial atención ${ }_{(25)}$.

Por último, es adecuado que se continúe investigando sobre la depresión como problema de salud en la población militar del país, a fin de identificar los determinantes de la enfermedad y priorizar las intervenciones, que coadyuven a la no afectación de la vida futura, en la reinserción a la comunidad y en el contexto familiar, una vez los soldados dejen la milicia ${ }_{(26)}$.

Una de las limitaciones principales en este estudio fue el período de aplicación de la prueba para determinar la depresión, en tanto fue suministrada en el primer mes de adaptación a la vida militar, etapa que presenta por sus características especiales desajustes psicológicos y emocionales ya que los jóvenes cambian sus estilos de vida y en el ejército hay actividades físicas y psicológicas que ponen a prueba sus capacidades de adaptación. Este proceso de adaptación por lo general causa llanto incontrolado, tristeza, sentimientos de soledad, angustia de separación, aunado a los procesos evolutivos del paso de la adolescencia a la vida adulta, lo que puede explicar por qué el grupo de mayor prevalencia en este estudio es el más joven.

\section{Limitaciones}

Una de las limitaciones fundamentales en este estudio se generó en el proceso de recolección de la información ya que en la fase de selección para la incorporación al servicio militar, los jóvenes que refieren consumo de sustancias psicoactivas no cumplen el perfil y no son incorporados, lo que conlleva a que algunos jóvenes mientan durantre la entrevista sobre su consumo, además, en la institución no se aplican pruebas de detección del consumo.

El grupo de psicólogas militares utilizó diversas estrategias que llevaran a los jóvenes a comprender la realidad y la importancia de aceptar su consumo como preservación de su integridad. Los jóvenes manifestaban querer estar en el ejército para intentar "salir del vicio". Sin embargo, el ejército se convierte en un entorno de riesgo que no posibilita la rehabilitación, antes bien, se puede incrementar por la exposición a niveles de estrés elevado. La limitación del estudio, al tratarse de un tema tan sensible éticamente, fue sorteada gracias al accionar de las profesionales de psicología militar, que, en algunos casos, debieron contactar con los familiares de los jóvenes consumidores para solicitar un tratamiento integral en las adicciones.

\section{Agradecimientos}

Al equipo de psicología militar y al batallón que permitió este estudio y a los jóvenes soldados que voluntariamente hicieron parte de este. En especial, se agradece a los oficiales que atentos a los resultados solicitaron el desacuartelamiento por tercer examen médico de los casos que fueron necesarios.

\section{Conflicto de intereses}

Los autores no manifiestan conflictos de intereses.

\section{Referencias}

1. Organización Mundial de la Salud [OMS]. Depresión [Internet]. 2018 [consultado 2019 Feb 5]. Disponible en: https://www.who.int/es/news-room/fact-sheets/ detail/depression

2. Sociedad Española de Psquiatria. Trastorno bipolar (maníaco depresivo) [Internet]. 2010 [consultado 2019 Feb 13]. Disponible en: http://www.sepsiq.org/ file/Royal/TRASTORNO BIPOLAR.pdf

3. Marcus M, Yasamy T, Ommeren M, Chisholm D, Shekhar S. Depresión: una crisis global. [Internet] 2012 [consultado 2019 Feb 6]. Disponible en: http://www. infocoponline.es/pdf/DMSaludMentalDepresion.pdf

4. Federación Mundial de la Salud Mental. Depresión: una crisis global día mundial de la salud mental [Internet] 2012 [consultado 2019 Jun 24]. Disponible en:http://www.infocoponline.es/pdf/ DMSaludMentalDepresion.pdf

5. Organización Mundial de la Salud. Depresión y otros trastornos mentales comunes Estimaciones sanitarias mundiales [Internet] 2017 [consultado 2019 Feb 5]. Disponible en: http://iris.paho.org/xmlui/bitstream/ handle/123456789/34006/PAHONMH17005-spa.pdf

6. Londoño Pérez C, González Rodríguez M. Prevalencia de depresión y factores asociados en hombres. Rev Acta Colomb Psicol [Internet]2016 [consultado 2019 Jun 24];19(2):p.315-29. Disponible en:http://editorial. ucatolica.edu.co/ojsucatolica/revistas_ucatolica/ index.php/acta-colombiana-psicologia/article/ view/1136/1144

7. Sanabria Ferrand PA, Guavita Falla PM. Prevalencia de sintomatología depresiva en una población estudiantil de la facultad de medicina de la universidad militar nueva granada, Bogotá Colombia. Rev. Fac. Med. Univ. Nac. Colomb. [Internet]. 2006 [consultado 2019 Feb 5]. Disponible en: http://www.scielo.org.co/ $\mathrm{pdf} / \mathrm{rfmun} / \mathrm{v} 54 \mathrm{n} 2 / \mathrm{v} 54 \mathrm{n} 2 \mathrm{a} 03 . \mathrm{pdf}$

8. El tiempo. cifras sobre depresion en colombia y en el mundo segun la OMS [Internet]. 2017 [consultado 2019 Feb 5]. Disponible en: https://www.eltiempo. $\mathrm{com} /$ vida/salud/cifras-sobre-depresion-en-colombiay-en-el-mundo-segun-la-oms-61454 
9. Ministerio de Salud y Protección Social. Boletín de salud mental depresión Bogotá. [Internet] 2017 [consultado 2019 Feb 5]. Disponible en: https://www. minsalud.gov.co/sites/rid/Lists/BibliotecaDigital/ RIDE/VS/PP/ENT/boletin-depresion-marzo-2017.pdf

10. Dirección general de sanidad militar. Plan maestro integral de salud mental para las fuerzas militares de Colombia. [Internet] 2012 [consultado 2019 Feb 5]. Disponible en: https://www.sanidadfuerzasmilitares. mil.co/la_entidad/dependencias/grupo_gestion_salud/ salud_mental/25199

11. Londoño C, Peñate W, González M. Validación Diferencial y Discriminante del Cuestionario de Depresión para Hombres (CDH). Rev Ter psicológica [Internet]. 2016 [consultado 2019 Jun 24];34(2):p.129-42. Disponible en: http://www. scielo.cl/scielo.php?script=sci_arttext\&pid=S0718$48082016000200005 \& \operatorname{lng}=$ en\&nrm=iso\&tlng=en

12. Collazos M V. Trastornos mentales y problemas de salud mental. Día Mundial de la Salud Mental. Rev Salud Ment. [Internet] 2007 [consultado 2019 Jun 24]; 30:p.75-80. Disponible en: http://www.redalyc.org/ pdf/582/58230211.pdf

13. Tobón C, Aguirre-Acevedo DC, Velilla L, Duque J, Ramos CP, Pineda D. Perfil psiquiátrico, cognitivo y de reconocimiento de características emocionales de un grupo de excombatientes de los grupos armados ilegales en Colombia. Rev Colomb Psiquiatr. [Internet] 2016 [consultado 2018 jun. 1]; 45(1):p.2836. Disponible en: http://www.scielo.org.co/pdf/rcp/ v45n1/v45n1a06.pdf

14. Jiménez WA. Salud mental en el posconflicto colombiano. Rev .Crim. [Internet]. 2009 [consultado 2018 Jun 1];51:p.179-92. Disponible en: http://www. scielo.org.co/pdf/crim/v51n1/v51n1a07.pdf

15. Adler A, Huffman A, Bliese P, Castro A. The Impact of Deployment Length and Experience on the WellBeing of Male and Female Soldiers. Rev. J. Occup. Health Psychol. [Internet] 2005 [consultado 2018 jun. 1]; 10:p.121-37. Disponible en: https://www.ncbi. nlm.nih.gov/pubmed/15826223

16. Browne T, Hull L, Horn O, Jones M, Murphy D, Fear $\mathrm{N}$, et al. Explanations for the increase in mental health problems in UK reserve forces who have served in Iraq. Rev Br J psychiatry. [Internet] 2007 [consultado 2018 Jun. 2];190:p.484-9. Disponible en: https:// www.ncbi.nlm.nih.gov/pubmed/17541107
17. Corzo PA. Trastorno por estrés postraumático en psiquiatría militar. Rev. MED. [Internet] 2009 [consultado 2018 Jun 10]; 17:P.81-6. Disponible en: http://www.scielo.org.co/scielo.php?script=sci_arttext \&pid=S0121-52562009000100012

18. Luque DA, García montaño E, García Roncallo P, Márquez Jiménez L, Niebles charris J, Pérez pedraza D. Perfil cognitivo y prevalencia de depresión, desesperanza y riesgo suicida en jóvenes vinculados al primer nivel de formación en una escuela militar colombiana. Rev. Psicología desde el Caribe. [Internet] 2011 [consultado 2018 Jun 9]; Disponible en: https:// psycnet.apa.org/record/2017-10789-006

19. Jiménez Villamizar MP, Linero Montaño L. Riesgo suicida, desesperanza y factores asociados en personal privado de la libertad de un Centro de Reclusión Militar en Colombia. Rev. Facultad de Salud. [Internet] 2011[consultado 2018 Jun 10];7485. Disponible en: http://www.scielo.org.co/pdf/psdc/v33n3/2011-7485psdc-33-03-00299.pdf

20. Moreno DSM. Prevalencia de depresión en los aspirantes a soldados de Inteligencia Militar. Rev la Fac Ciencias Médicas. [Internet] 2008 [consultado 2018 Jun 10];33:p.24-8. Disponible en: http://www.imbiomed.com.mx/1/1/articulos. php?method=showHomeMagazine\&id_revista=203

21. Grieger TA, Cozza SJ, Urzano RJ, Hoge C, Martínez PE, Engel CC, et al. Posttraumatic Stress Disorder and Depression in Battle-Injured Soldiers. Rev Am J Psychiatry. [Internet] 2006 [consultado 2018 Jun 10]; 163:p.1777-83. Disponible en: https://www.ncbi.nlm. nih.gov/pubmed/17012689

22. Abello Luque D, Márquez Jiménez L, Niebles Charris J, Pérez Pedraza D. Perfil cognitivo y prevalencia de depresión, desesperanza y riesgo suicida en jóvenes vinculados al primer nivel de formación en una escuela militar colombiana. Rev. Psicol desde el Caribe [Internet] 2016 [consultado 2018 Jun 11]; 33:p.299-311. Disponible en: http://www.scielo.org. co/pdf/psdc/v33n3/2011-7485-psdc-33-03-00299.pdf

23. Shelef L, Korem N, Yavnai N, Yedidya R, Ginat $\mathrm{K}$, Shahar G, et al. Depression and impulsiveness among soldiers who died by suicide: a psychological autopsy study. Rev. affective disorder. [Internet]. 2018 [consultado 2018 Jun.10] Disponible en: https://www. ncbi.nlm.nih.gov/pubmed/29665517

24. Kim NY, Lee PK, Lim MH. Kim, N. Y., Lee, P. K., \& 
Lim, M. H. Suicidal Idea,ADHD, Depression, Anxiety, Self-esteem and Impulsiveness in Korean Soldiers. Rev Journal of Psychiatry. [Internet] 2015 [consultado 2018 Jun 18];18:p.314. Disponible en: https://www. omicsonline.org/open-access/suicidal-idea-adhddepression-anxiety-selfesteem-and-impulsiveness-inkorean-soldiers-2378-5756-1000314.pdf

25. Delgado N. Aproximación conceptual de la psicología militar. Rev Bachelor's thesis, Fac Psicol. [Internet] 2016[consultado 2018 Jun 18] Disponible en: https:// repository.javeriana.edu.co/handle/10554/21334

26. Jiménez W. Salud mental en el postconflicto colombiano. Rev Crim [Internet]. 2009; [consultado 2018 Jun 18]; 51:p.179-93. Disponible en: http:// www.policia.gov.co/imagenes_ponal/dijin/revista_ criminalidad/vol51_1/08salud.pdf 